\title{
First-principles investigation of anisotropic constitutive relationships in pentaerythritol tetranitrate
}

\author{
M. W. Conroy, ${ }^{1}$ I. I. Oleynik, ${ }^{1}$ S. V. Zybin, ${ }^{2}$ and C. T. White ${ }^{3}$ \\ ${ }^{1}$ Department of Physics, University of South Florida, Tampa, Florida 33620, USA \\ ${ }^{2}$ Materials and Process Simulation Center, California Institute of Technology, Pasadena, California 91125, USA \\ ${ }^{3}$ Naval Research Laboratory, Washington, DC 20375, USA \\ (Received 21 November 2007; published 7 March 2008)
}

\begin{abstract}
First-principles density functional theory (DFT) calculations have been used to obtain the constitutive relationships of pentaerythritol tetranitrate (PETN-I), a crystalline energetic material. The isotropic equation of state (EOS) for hydrostatic compression has been extended to include uniaxial compressions in the $\langle 100\rangle$, $\langle 010\rangle,\langle 001\rangle,\langle 110\rangle,\langle 101\rangle,\langle 011\rangle$, and $\langle 111\rangle$ crystallographic directions up to a compression ratio of $V / V_{0}$ $=0.70$. DFT predicts equilibrium properties such as lattice parameters and elastic constants, as well as the hydrostatic EOS, in agreement with available experimental data. Our results show a substantial anisotropy of various properties of PETN-I upon uniaxial compression. To characterize the anisotropic traits of PETN, different physical properties of the uniaxially compressed crystal such as the energy per atom, band gap, and stress tensor have been evaluated as a function of compression ratio. The maximum shear stresses were calculated and examined for a correlation with the anisotropy in shock-initiation sensitivity.
\end{abstract}

DOI: 10.1103/PhysRevB.77.094107

PACS number(s): 62.50.-p, 62.50.Ef, 64.30. $-\mathrm{t}, 71.15 . \mathrm{Nc}$

\section{INTRODUCTION}

One of the goals of energetic materials (EMs) research is to investigate the fundamental physical and chemical properties of EM molecular crystals from first principles based on the underlying atomic scale structure. Accurate equations of state (EOS) are of particular interest because they establish fundamental relationships between thermodynamic variables and provide necessary input for the description of materials at the mesoscopic and continuum levels. Ultimately, these EOS are governed by interactions at the atomic scale, which provides an excellent opportunity for atomic-scale simulation techniques to link the microscopic and macroscopic properties of a material.

Most of the theoretical and experimental investigations focus on isotropic EOS, i.e., relationships between pressure and volume. However, due to the dominant role of shockinduced phenomena during the initiation and propagation of detonation, there is an urgent need to extend the isotropic EOS to include the response of EMs upon anisotropic uniaxial compressions.

Pentaerythritol tetranitrate (PETN), an important EM, has attracted considerable interest from the EM community because of the discovery of its anisotropic shock sensitivity by Dick. ${ }^{1-3}$ Some prior work on PETN has indicated anomalous behavior upon shock compression. In particular, Halleck and Wackerle ${ }^{4}$ performed shock compressions of up to about 4 GPa on single-crystal samples with planes cut perpendicular to the $\langle 110\rangle$ and $\langle 001\rangle$ directions. For shocks at and above 4 GPa on the $\langle 110\rangle$ crystals, shock-induced decomposition was expected from a rapid increase in stress amplitude approximately $0.3 \mu$ s after shock arrival. However, it was Dick who unambiguously demonstrated the anisotropy in shock sensitivity through his classic experiments. He found that when compressed in its most sensitive direction, $\langle 110\rangle$, detonation has been observed ${ }^{3}$ in PETN crystals under shock stresses as small as $4.2 \mathrm{GPa}$ [from Dick, ${ }^{3}\langle h k l\rangle$ denotes the direction perpendicular to the $(h k l)$ plane]. The $\langle 001\rangle$ direction has also been reported as sensitive. ${ }^{1-3}$ However, PETN is very insensitive to shocks in the $\langle 100\rangle$ direction ${ }^{1-3,5}$ and the $\langle 101\rangle$ direction has also been found to be relatively insensitive. ${ }^{1,3}$ Later, the anisotropic shock sensitivity of PETN was also investigated by Soulard. ${ }^{6,7}$

There have also been a number of works suggesting mechanisms for the directional dependence of sensitivity in PETN. Dick et al. ${ }^{1-3}$ have proposed a model based upon steric hindrance to shear, which suggests that sensitivity is related to the ease by which slip is allowed via the available slip systems under shock compression. Further, Jindal and Dlott ${ }^{8}$ have suggested that anisotropic heating in response to shock compression might be responsible for directional dependence of sensitivity. Gruzdkov and Gupta ${ }^{9}$ have added to the possible mechanisms by arguing that changes in the local polarization of the lattice arise due to conformational changes in PETN molecules under compression, allowing decomposition via ionic reactions.

The macroscopic properties of several energetic materials including PETN, cyclotetramethylene tetranitramine (HMX), cyclotrimethylene trinitramine (RDX), and triaminotrinitrobenzene (TATB) have been studied and described well. ${ }^{10,11}$ However, the microscopic behavior responsible for the shock initiation observed on the macroscopic scale is not well understood. First-principles simulation of these materials at the atomic and molecular level thus provides an excellent opportunity for the investigation of microscopic processes leading to shock initiation. Because of the relatively large unit cells and complex geometries of these energetic materials, firstprinciples calculations have only been feasible for the last decade due to both advances in computational methodology and the exponential growth in computational power of modern computers. PETN is an exemplary EM for these calculations, as its anomalous anisotropic shock-initiation behavior $^{1-3}$ can be probed at the atomic scale.

The crystal structure of PETN was studied experimentally via $\mathrm{x}$-ray diffraction by Booth and Llewellyn, ${ }^{12}$ whose data 
were later revised by Trotter. ${ }^{13} \mathrm{~A}$ tetragonal lattice with space-group symmetry $P \overline{4} 2_{1} c$ was determined, with two $\mathrm{C}\left(\mathrm{CH}_{2} \mathrm{ONO}_{2}\right)_{4}$ molecules (58 atoms) per unit cell. ${ }^{12}$ Importantly, it was noted that weak van der Waals forces play a vital role in the intermolecular interactions. ${ }^{12}$ We denote the PETN-I polymorph as PETN, but a second polymorph, PETN-II, has been reported at high temperature ${ }^{14}$ and a third polymorph, PETN-III has recently been reported near 6 GPa. ${ }^{15}$ In addition, Olinger et al. ${ }^{16}$ performed x-ray diffraction experiments of volume compression of PETN up to 10 $\mathrm{GPa}$ at room temperature.

Several theoretical studies of energetic materials including PETN may be found in the literature. ${ }^{17-29}$ Of particular importance for comparison with this work are the firstprinciples studies by Gan et al., ${ }^{19}$ Byrd and Rice, ${ }^{24}$ and Brand. ${ }^{20}$ Gan et al. ${ }^{19}$ performed density functional theory (DFT) calculations using the Perdew-Burke-Ernzerhof (PBE) functional and a Gaussian basis set to simulate the hydrostatic compression of PETN. Byrd and Rice ${ }^{24}$ have performed a comprehensive study of the accuracy of DFT in application to several EMs. They used DFT with a planewave basis set and the Perdew-Wang (PW91), PBE, and local density approximation (LDA) flavors of density functional theory at various cutoff energies. Also, Brand ${ }^{20}$ performed Hartree-Fock calculations of hydrostatic compression with a Gaussian basis set.

Most of the calculations deal with equilibrium properties or hydrostatic compression. Therefore the goal of this work is to extend the isotropic EOS for PETN to study its anisotropic properties upon high compression. The uniaxial compression studies, motivated by the highly anisotropic shocksensitivity behavior of the crystal, were performed to investigate relative changes of physical properties upon uniaxial compression along seven low index crystallographic directions: $\langle 100\rangle,\langle 010\rangle,\langle 001\rangle,\langle 110\rangle,\langle 101\rangle,\langle 011\rangle$, and $\langle 111\rangle$. We also completed studies of the equilibrium properties and the hydrostatic EOS of PETN-I for the purpose of evaluating the accuracy of DFT as applied to EM crystals and for comparison with previous calculations.

The shear stresses upon uniaxial compression are of particular interest because they are usually considered to be the driving forces of plastic deformations in crystals and the sources of mechanochemical reactions behind the shock wave front. Therefore we investigate the behavior of shear stresses in PETN upon uniaxial compression with the specific aim of seeing if there is any correlation with observed anisotropic shock sensitivity.

\section{COMPUTATIONAL DETAILS}

First-principles DFT $^{30}$ calculations were performed using the Vienna ab initio simulation package ${ }^{31,32}$ (VASP). To obtain accurate results, tests were performed to choose the appropriate parameters that control the quality of the calculations: the DFT functional, pseudopotential, $k$-point set, and energy cutoff. The DFT functionals tested were PW9133,34 and $\mathrm{PBE}^{35,36}$ of the DFT generalized gradient approximation (GGA). VASP employs either ultrasoft pseudopotentials ${ }^{37,38}$ (USP) or the projector-augmented wave ${ }^{39,40}$ (PAW) method to eliminate explicit treatment of the core electrons during self-consistent electronic structure calculations. Therefore the PW91 functional was tested with USP potentials, and both the PW91 and PBE functionals were tested with PAW potentials. For each, the experimental structure of PETN was relaxed with an energy cutoff of $1000 \mathrm{eV}$ and a $2 \times 2 \times 2$ Monkhorst-Pack ${ }^{41}$ (MP) $k$-point grid. We found that the PBE functional and the PAW potential produced better agreement with experiment than any other combination of functional and potential.

The energy cutoff is a very important parameter that controls the completeness of the plane-wave basis set. Together with the density of $k$-point sampling of the Brillouin zone, it determines the numerical accuracy of DFT plane-wave calculations. It is also known that the DFT calculations of molecular crystals, which are characterized by a highly inhomogeneous distribution of electron density, require larger basis sets to sample the very small electron density in the intermolecular space of the crystal. Therefore extensive tests were performed to choose the energy cutoff and $k$-point density that would provide sufficient accuracy in the calculation of total energies, atomic forces, and stresses acting on the PETN crystal both at equilibrium and at highly compressed states.

First, total energy calculations without relaxation were performed on both the experimental structure and a halfcompressed structure using an energy cutoff of $1000 \mathrm{eV}$ to test for a sufficient $k$-point set. The half-compressed structure was made by scaling the lattice parameters by $0.5^{1 / 3}$, and it was used in this test to be sure that, as the Brillouin zone enlarged during compression, the fixed $k$-point set would still provide results with sufficient accuracy. We found that the $2 \times 2 \times 2$ MP set yielded convergence results better than $0.0007 \mathrm{eV} /$ atom in energy, $0.02 \mathrm{eV} / \AA$ in forces, and 0.05 $\mathrm{GPa}$ in pressure, and was chosen for the equilibrium structure and hydrostatic compression calculations. It should be noted that the $k$-point spacing in this grid corresponded to an average $k$-point spacing of $0.08 \AA^{-1}$ in each direction when the structure was scaled to half of its experimental volume. Energy cutoff values of 400, 500, 700, and $1000 \mathrm{eV}$ were then tested on the experimental structure via total energy calculations using the PBE functional, the PAW pseudopotentials, and the $2 \times 2 \times 2 \mathrm{MP}$ grid. The minimum energy cutoff, and thus basis set size, with sufficient accuracy was desired. From this comparison, the $700 \mathrm{eV}$ energy cutoff was chosen for all later calculations. This cutoff yielded convergence better than $0.4 \mathrm{GPa}$ in pressure, $0.0015 \mathrm{eV} /$ atom in energy, and $0.015 \mathrm{eV} / \AA$ in forces. It should be noted that $700 \mathrm{eV}$ is almost twice the nominal energy cutoff for the PAW potential used, $400 \mathrm{eV}$.

To obtain the theoretical cell parameters for PETN, the experimental structure was relaxed using the parameters above via the quasi-Newton structure minimization method as implemented in VASP. The unit cell size and shape, as well as the atomic coordinates, were allowed to simultaneously relax without a constraint on symmetry. The convergence criterion for electronic steps for all calculations was set to $10^{-6} \mathrm{eV}$, whereas ionic steps were stopped when the maximum force on any atom was less than $0.01 \mathrm{eV} / \AA$. Hydrostatic compression of PETN was simulated by VASP calcula- 
TABLE I. Calculated equilibrium structure of PETN compared with experiment.

\begin{tabular}{ccccc}
\hline \hline Work & $\begin{array}{c}a \\
(\AA)\end{array}$ & $\begin{array}{c}b \\
(\AA)\end{array}$ & $\begin{array}{c}c \\
(\AA)\end{array}$ & $\begin{array}{c}V \\
\left(\AA^{3}\right)\end{array}$ \\
\hline $\begin{array}{l}\text { Booth } \text { et al. } \\
\text { (experiment }) \\
(\text { Ref. } 12)\end{array}$ & 9.38 & 9.38 & 6.70 & 589.5 \\
This work & $9.617(+2.5 \%)$ & $9.612(+2.5 \%)$ & $6.826(+1.9 \%)$ & $630.99(+7.0 \%)$ \\
\hline \hline
\end{tabular}

tions that compressed the unit cell from $V / V_{0}=1.00$ to 0.60 in increments of 0.02 , where $V_{0}$ is the predicted volume at zero pressure. At each compression step, the unit cell shape and atomic coordinates were allowed to relax under constant volume without symmetry constraints. The maximum force on any atom was less than $0.03 \mathrm{eV} / \AA$ for all compression simulations.

Uniaxial compressions were performed along seven low index crystallographic directions: $\langle 100\rangle,\langle 010\rangle,\langle 001\rangle,\langle 110\rangle$, $\langle 101\rangle,\langle 011\rangle$, and $\langle 111\rangle$. For each compression direction, the Cartesian coordinate system, used to specify components of the lattice vectors, was rotated to orient the $x$ axis along the specific crystallographic direction to be compressed. Then, the $x$ component of each lattice vector was scaled by increments of $2 \%$ up to $V / V_{0}=1.06$ and down to $V / V_{0}=0.70$. The uniaxial expansions and compressions near equilibrium were also used to obtain elastic constants of PETN. At each step, only the atomic coordinates were allowed to relax while the unit cell was held fixed. To avoid any jumps in physical quantities due to insufficient sampling of the Brillouin zone, the MP $k$-point set was generated for each uniaxial compression to ensure an average grid spacing of $0.08 \AA^{-1}$ in each direction when the structure was scaled to half of its experimental volume along the corresponding compression direction.

\section{EQUILIBRIUM PROPERTIES AND HYDROSTATIC EOS}

The structure of PETN-I at zero pressure was calculated, and the unit cell parameters were compared with the data refined by Trotter $^{13}$ from the experiments of Booth and Llewellyn, ${ }^{12}$ see Table I. The percent error between our cal-

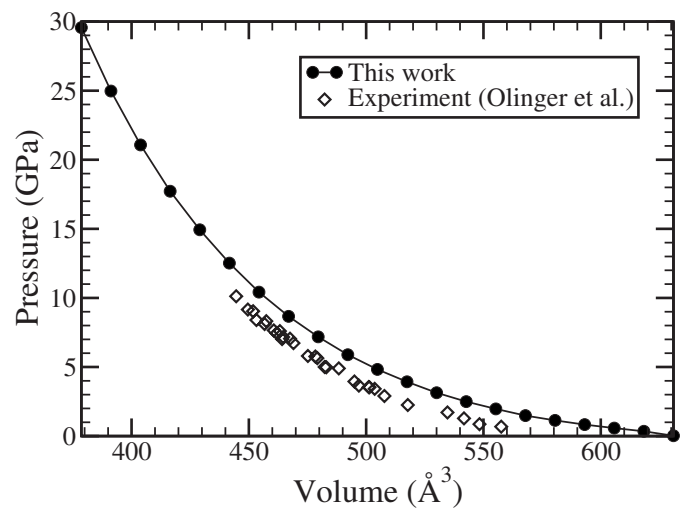

FIG. 1. Isothermal hydrostatic EOS of PETN-I. culations of equilibrium lattice constants and experiment is about $2 \%$ to $3 \%$. As in previous DFT studies of energetic materials, ${ }^{18,22}$ the prediction of greater lattice constants was believed to be due to an inadequate description of van der Waals dispersive interactions in DFT functionals. Predicted lattice angles, similar to experiment, ${ }^{12,13}$ were calculated to be $90.0^{\circ}$.

The PETN-I isothermal equation of state was obtained by performing DFT hydrostatic compression simulations at $0 \mathrm{~K}$. Figure 1 compares the DFT results with the roomtemperature experimental data from the work of Olinger, Halleck, and Cady. ${ }^{16}$ The range in volume changes shown is from $60 \%$ to $100 \%$ of the calculated equilibrium volume, $630.99 \AA^{3}$. At $V / V_{0}=0.60$, the calculated pressure is approximately $30 \mathrm{GPa}$, which is also approximately the detonation pressure of PETN. ${ }^{42}$ The calculated pressure at a given volume is higher than the experimental value, which is believed to be due to the inadequate description of weak van der Waals dispersive interactions by DFT. ${ }^{22}$ In this regard, the discrepancy between the experimental and calculated isotherms is reduced as volume decreases, which is probably due to increased overlap of the electron densities of neighboring molecules, leading to a better description of the intermolecular interactions by DFT. This trend has been observed previously in hydrostatic compression studies of energetic molecular crystals using DFT. ${ }^{22}$

The lattice constants $a$ and $c$ as a function of pressure are compared with experimental data ${ }^{16}$ in Fig. 2. As pressure increases, the experimental value of $a$ approaches the calculated value. However, the calculated value of $c$ is greater than the experimental value for all pressures. This trend has been observed before in the DFT calculations of Brand. ${ }^{20}$

Bond lengths of a PETN molecule within the crystal were plotted as a function of $V / V_{0}$ in Fig. 3. As observed in a prior

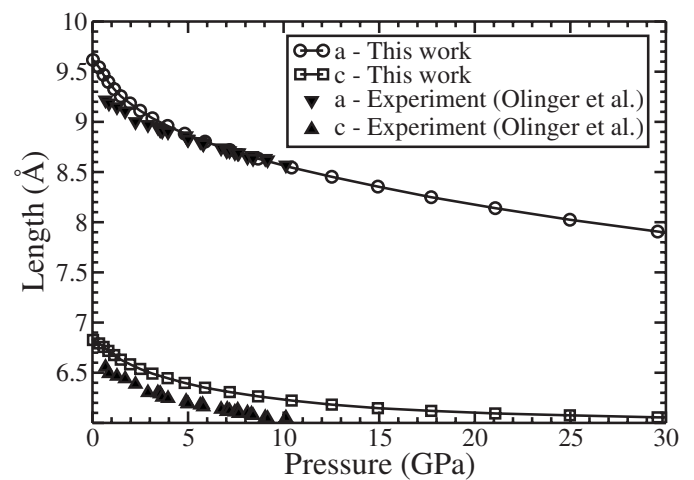

FIG. 2. Lattice parameters of PETN under hydrostatic compression. 

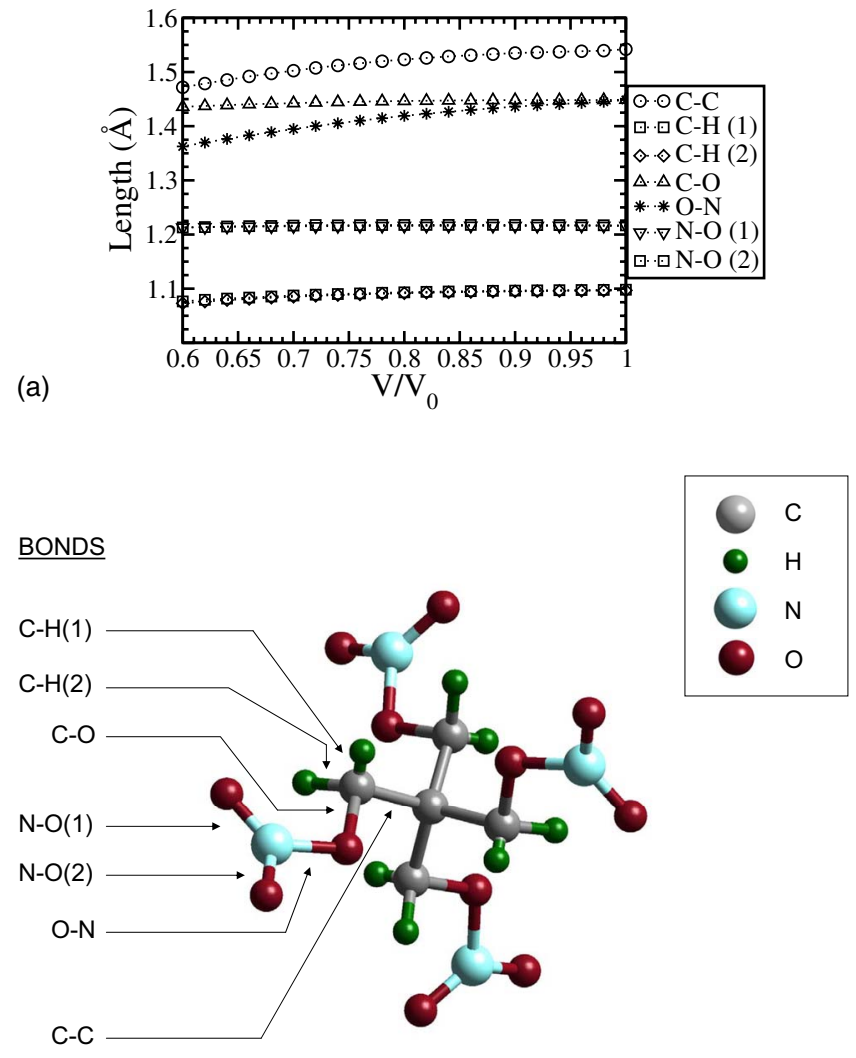

(b)

FIG. 3. (Color online) Bond lengths of PETN under hydrostatic compression. The bonds within a molecule are indicated on the right.

DFT study by Gan, Sewell, and Challacombe, ${ }^{19}$ the C-C bond and the $\mathrm{O}-\mathrm{N}$ bond experience greater changes than the other bonds. The greatest change in bond length observed was for the proximal $\mathrm{O}-\mathrm{N}$ bond, which decreased by $0.086 \AA$.

The bulk modulus, $B_{0}=-V(d P / d V)$, and its derivative with respect to pressure, $B_{0}^{\prime}$, were obtained from fitting the PETN-I EOS data to the Birch-Murnaghan EOS, ${ }^{19}$

$$
P=\frac{3}{2} B_{0}\left(\eta^{-7 / 3}-\eta^{-5 / 3}\right)\left[1+\frac{3}{4}\left(B_{0}^{\prime}-4\right)\left(\eta^{-2 / 3}-1\right)\right],
$$

where $\eta=V / V_{0}$, using pressures from about $-0.3-10.4 \mathrm{GPa}$ ( $\left.V / V_{0}=1.06-0.72\right)$. Following the work of Gan, Sewell, and Challacombe, ${ }^{19}$ we have restricted our fitting to pressures below $10.45 \mathrm{GPa}$ to be consistent with the experimental work of Olinger et al. ${ }^{16}$ Table II shows our data as compared with the Birch-Murnaghan fitting ${ }^{19}$ of the experimental data obtained by Olinger et al. ${ }^{16}$ and the bulk modulus obtained via experimental elastic constants, ${ }^{43}$

$$
B=\frac{C_{33}\left(C_{11}+C_{12}\right)-2 C_{13}^{2}}{\left(C_{11}+C_{12}\right)+2 C_{33}-4 C_{13}},
$$

from Winey and Gupta. ${ }^{44}$ Not included in Table II are the values of the bulk modulus from the theoretical works of Gan et al. $\left(B_{0}=14.5 \mathrm{GPa}, B_{0}^{\prime}=6.7\right)$ which were obtained by fitting their EOS data to the Birch-Murnaghan EOS (1) and

\begin{tabular}{|c|c|c|}
\hline Work & $\begin{array}{c}B_{0} \\
(\mathrm{GPa})\end{array}$ & $B_{0}^{\prime}$ \\
\hline $\begin{array}{c}\text { This work } \\
\text { (Birch-Murnaghan) }\end{array}$ & 9.1 & 8.3 \\
\hline $\begin{array}{c}\text { Olinger } \text { et al. } \\
\text { (Birch-Murnaghan) } \\
\text { (Ref. 16) }\end{array}$ & 9.4 & 11.3 \\
\hline $\begin{array}{l}\text { Winey and Gupta } \\
\text { (Ref. 44) }\end{array}$ & 9.1 & - \\
\hline
\end{tabular}

TABLE II. Bulk modulus results.

Brand $^{45}$ obtained $B_{0}=9.38$ GPa by using elastic constants via Eq. (2).

The bulk modulus can be found by fitting to other equations, such as the Murnaghan equation and the Hugoniot conservation equations used by Gan et al. ${ }^{19}$ The values of the bulk modulus found by fitting our data set to these equations were within about $10 \%$ of the value obtained by fitting to Eq. (1), similar to the results obtained by Gan et al. ${ }^{19}$

Using the uniaxial compression data discussed below, the energy $E$ of the crystal as a function of strain $\epsilon$ was fit to a fourth-order polynomial $E(\epsilon)$. The elastic constants calculated were then found from the relation

$$
C_{i i}=\frac{1}{V_{0}}\left(\frac{\partial^{2} E}{\partial \epsilon_{i i}^{2}}\right),
$$

where $i=1,2,3$. The calculated values for these elastic constants are in good agreement with the experimental data analyzed by Winey and Gupta, ${ }^{44}$ see Table III.

\section{UNIAXIAL COMPRESSIONS}

The diagonal elements of the stress tensor, $\sigma_{x x}, \sigma_{y y}$, and $\sigma_{z z}$, as a function of compression ratio from $V / V_{0}=0.70-1.00$ for each uniaxial compression are shown in Figs. 4(a)-4(c), respectively. The anisotropic character of the constitutive relationships is clearly seen from Fig. 4(a), which shows substantial differences in longitudinal stresses between uniaxial compressions along different crystallographic directions as compared with pressure, $P=\sigma_{x x}=\sigma_{y y}=\sigma_{z z}$, from hydrostatic compressions. For strains below $15 \%$, the calculations indicate that the crystal is less compressible in the $\langle 011\rangle$ and $\langle 101\rangle$ directions and more compressible in the $\langle 110\rangle$ and $\langle 001\rangle$ directions. However, for strains greater than $25 \%$, the $\langle 001\rangle$ and $\langle 110\rangle$ directions emerge as the least compressible. Based upon the work of Jindal and Dlott, ${ }^{8}$ the anisotropic

TABLE III. Elastic constants of PETN.

\begin{tabular}{cccc}
\hline \hline Work & $\begin{array}{c}C_{11} \\
(\mathrm{GPa})\end{array}$ & $\begin{array}{c}C_{22} \\
(\mathrm{GPa})\end{array}$ & $\begin{array}{c}C_{33} \\
(\mathrm{GPa})\end{array}$ \\
\hline $\begin{array}{c}\text { This work } \\
\begin{array}{c}\text { Winey and Gupta } \\
\text { (Ref. 44) }\end{array}\end{array}$ & 18.3 & 18.5 & 14.2 \\
(17.22 & 17.22 & 12.17 \\
\hline
\end{tabular}



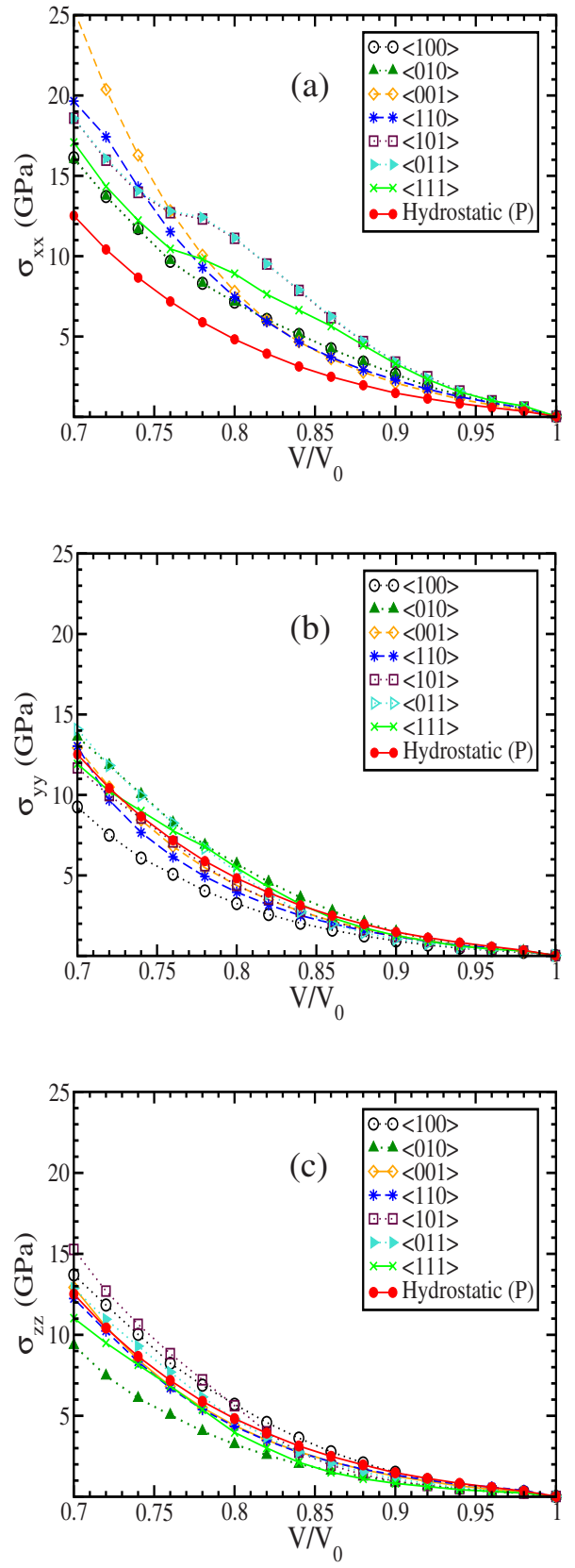

FIG. 4. (Color online) Stress tensor components $\sigma_{x x}(\mathrm{a}), \sigma_{y y}(\mathrm{~b})$, and $\sigma_{z z}$ (c) of PETN under uniaxial compression. The stresses for the hydrostatic compression are included for comparison to show the degree of anisotropy upon uniaxial compression.

compressibility of PETN could be an important factor for determining if anisotropic heating upon shock compression plays a significant role in its anisotropic detonation sensitivity. However, we do not calculate the Gruneisen parameters necessary for such a prediction. ${ }^{8}$

For shocks near $4 \mathrm{GPa}$ in the $\langle 110\rangle$ direction, evidence of detonation has been shown by experiment. ${ }^{3}$ Therefore it is likely that the anisotropic constitutive relationships cannot be probed experimentally when compressed in this direction beyond $4 \mathrm{GPa}$. However, the $\langle 100\rangle$ direction has been shown to be very insensitive, ${ }^{1-3,5}$ and therefore it is likely that the experimental dependence of shear stress on strain can be ob-

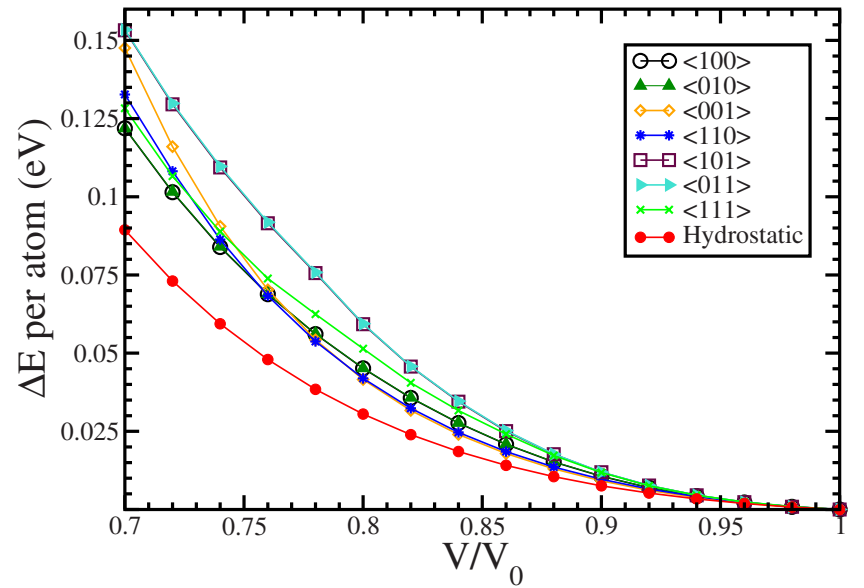

FIG. 5. (Color online) Change in energy per atom upon uniaxial and hydrostatic compressions.

tained for this direction in the compression interval shown in Fig. 4.

The change in energy per atom and the band gap of PETN-I as a function of compression for each uniaxial compression and the hydrostatic compression are shown in Figs. 5 and 6, respectively. Anisotropic behavior is clearly exhibited in both graphs. The greatest change in energy is experienced when the crystal is compressed in the $\langle 101\rangle$ or $\langle 011\rangle$ directions, and all uniaxial compressions raise the energy of the crystal more than hydrostatic compression because of the constraints of the unit cell in transverse directions. Similarly, the greatest reduction in the band gap is observed for the $\langle 101\rangle$ and $\langle 011\rangle$ uniaxial compressions. The smallest change in band gap is observed for hydrostatic compression, but it should be noted that the most sensitive direction, $\langle 110\rangle$, experiences only a slightly larger reduction in band gap than the hydrostatic compression.

\section{SHEAR STRESSES}

The shear stresses could be of importance in understanding the shock-induced anisotropy of EMs. The shock-loading conditions are characterized by fast uniaxial compression of the crystal along a specific crystallographic direction, i.e., the

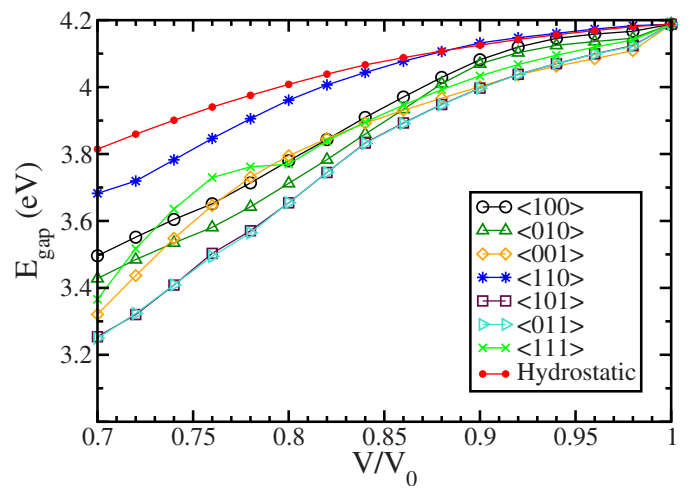

FIG. 6. (Color online) Band gap upon uniaxial and hydrostatic compression. 

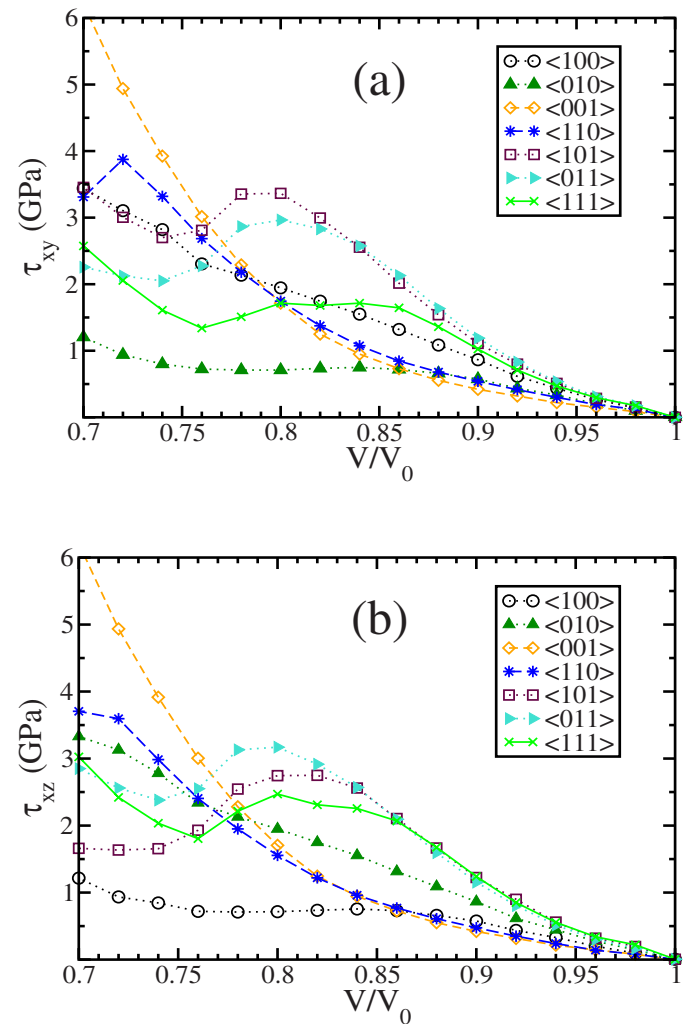

FIG. 7. (Color online) Shear stress maxima $\tau_{x y}$ (a) and $\tau_{x z}$ (b).

direction of shock-wave propagation. The shear stresses are the driving forces of shear-induced plastic deformations and mechanochemical events behind the shock-wave front. Therefore we calculate the maximum shear stresses for each uniaxial compression, see Figs. 7(a) and 7(b). Under the uniaxial compression, the crystal experiences the maximum shear stresses directed at $45^{\circ}$ to the compression direction, their values being $\tau_{x y}=\frac{1}{2}\left(\sigma_{x x}-\sigma_{y y}\right)$ and $\tau_{x z}=\frac{1}{2}\left(\sigma_{x x}-\sigma_{z z}\right)$.

The maximum shear stresses $\tau_{x y}$ and $\tau_{x z}$ for each compression are significantly larger than the values for $\tau_{y z}$, and therefore only the behavior of $\tau_{x y}$ and $\tau_{x z}$ is discussed below. For compressions greater than $V / V_{0}=0.76$, the more sensitive directions, $\langle 110\rangle$ and $\langle 001\rangle$, show greater magnitude of shear stresses $\tau_{x y}$ and $\tau_{x z}$ than the insensitive directions, $\langle 100\rangle$ and $\langle 101\rangle$. Based upon the greater shear stresses displayed for compression in the more sensitive directions, we have previously suggested a correlation between sensitivity and shear stress behavior under uniaxial compression. ${ }^{46-48}$ Here, we also examine the shear stress maxima, $\tau_{x y}$ and $\tau_{x z}$, as a function of longitudinal stress, $\sigma_{x x}$, and examine the possibility of a correlation between experimental sensitivity data and shear stresses in PETN, see Figs. 8(a) and 8(b). Note that each curve terminates at $V / V_{0}=0.70$. Both shear stress maxima for the sensitive directions, $\langle 110\rangle$ and $\langle 001\rangle$, are greater than the shear stress maxima for the insensitive direction, $\langle 100\rangle$, at longitudinal stresses greater than $4 \mathrm{GPa}$. Similarly, the shear stress maxima of the sensitive directions are greater than the other relatively insensitive direction, $\langle 101\rangle$, above $12 \mathrm{GPa}$. As stated above, $\langle 110\rangle$ compressions have experi-
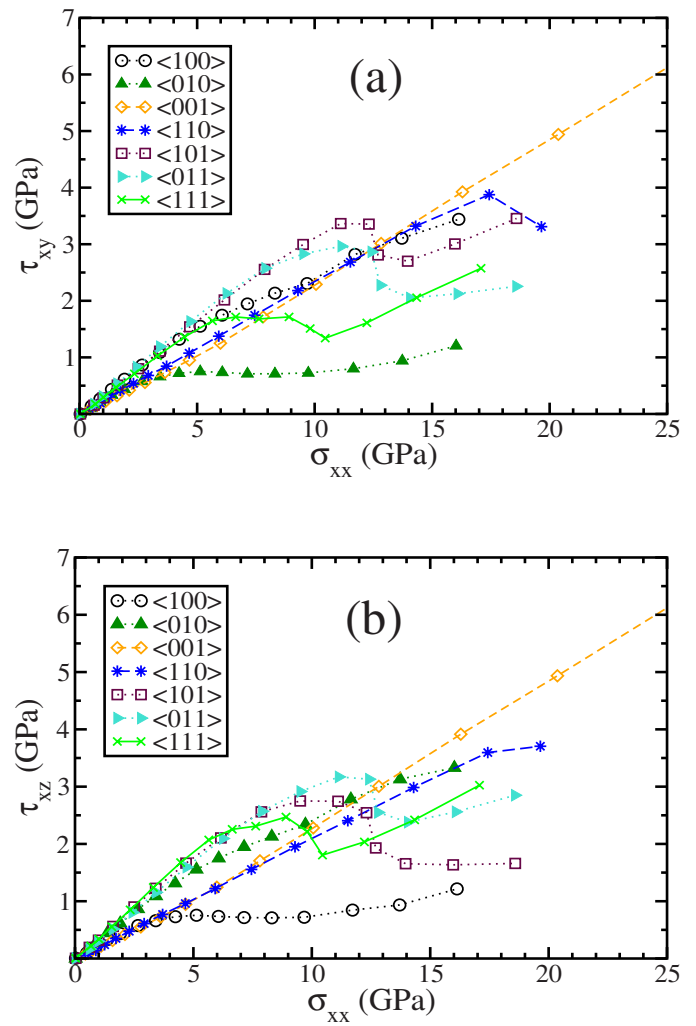

FIG. 8. (Color online) Shear stress maxima $\tau_{x y}$ (a) and $\tau_{x z}$ (b) as a function of longitudinal stress $\sigma_{x x}$.

enced a transition to detonation in Dick's experiment upon compression by longitudinal stresses as low as 4.2 GPa (Ref. 3) while $\langle 100\rangle$ shocks do not lead to detonation up to $\sim 19 \mathrm{GPa}$. Further, compressions of PETN in the $\langle 001\rangle$ direction have produced detonation at longitudinal stresses just above $12 \mathrm{GPa}$ (Ref. 3). The values of $\tau_{x y}$ and $\tau_{x z}$ for the $\langle 001\rangle$ compression do emerge as the greatest values for the compressions studied beyond $12 \mathrm{GPa}$, while below this value both shear stress maxima for $\langle 101\rangle$ compression remain the greatest. However, in Dick's experiment, shock in $\langle 101\rangle$ does not lead to detonation for the input stresses up to $\sim 15 \mathrm{GPa}$, though it exhibits an intermediate velocity transition (abrupt increase of the shock velocity and stress) at $8.8 \mathrm{GPa}$. Thus while there are some indications of a correlation between maximum shear stresses and shock sensitivity for the $\langle 100\rangle$, $\langle 110\rangle$, and $\langle 001\rangle$ cases, additional analysis needs to be performed. In particular, as suggested in the work of Dick, ${ }^{1}$ it might be helpful to consider the projection of shear stress maxima on available slip systems under uniaxial compression.

\section{CONCLUSIONS}

First-principles simulations of hydrostatic compression and uniaxial compressions in the $\langle 100\rangle,\langle 010\rangle,\langle 001\rangle,\langle 110\rangle$, $\langle 101\rangle,\langle 011\rangle$, and $\langle 111\rangle$ directions of PETN have been performed using DFT with the PBE functional and the PAW potential. The calculated lattice parameters were overestimated by about $3 \%$, which is believed to be due to a poor 
description of van der Waals dispersive interactions in the functionals of DFT. The calculated bulk modulus and elastic constants are in reasonable agreement with experiment. With the application of hydrostatic pressure, the calculated unit cell volume and lattice parameters of PETN show increasing agreement with experimental data.

The energy per atom, band gap, and stresses were calculated for each uniaxial compression and compared with the calculated results from hydrostatic compression. Our results show a substantial anisotropy of various properties of PETN-I upon uniaxial compressions. The maximum shear stresses were calculated and examined for a correlation with the anisotropy in shock-initiation sensitivity. The calculated maximum shear stresses, $\tau_{x y}$ and $\tau_{x z}$, upon compression in the sensitive $\langle 110\rangle$ and $\langle 001\rangle$ directions are greater than for the insensitive $\langle 100\rangle$ direction at longitudinal stresses above $4 \mathrm{GPa}$, but no clear correlation between maximum shear stresses and sensitivity is observed for the $\langle 101\rangle$ direction up to $12 \mathrm{GPa}$. In the future, we will perform a more detailed analysis of the shear stress behavior under uniaxial compressions by considering available slip systems of the PETN crystal upon compression in specific crystallographic directions, as suggested by the work of Dick, ${ }^{1}$ to establish more certain correlations with the anisotropic shock sensitivity of PETN.

\section{ACKNOWLEDGMENTS}

The work at USF was supported by the Office of Naval Research (ONR) through the Naval Research Laboratory (NRL) (Grants No. N00173-06-1-G022 and No. N00173-082-C002) and partly by the Army Research Office through the Multi-University Research Initiative on Insensitive Munitions (Grant No. W901NF-05-1-0266). The work at Caltech was supported by the Office of Naval Research (ONR) (Grant No. N00014-05-1-0778) and the Army Research Office through the Multi-University Research Initiative on Insensitive Munitions (Grant No. W911NF-05-1-0345). The work at NRL was supported by ONR both directly (Grant No. N00014-08-WX-20138) and through NRL. The computations were performed using NSF Teragrid computational facilities (Grant No. TG-DMR070018N).
${ }^{1}$ J. J. Dick, Appl. Phys. Lett. 44, 859 (1984).

${ }^{2}$ J. J. Dick, J. Appl. Phys. 81, 601 (1997).

${ }^{3}$ J. J. Dick, R. N. Mulford, W. J. Spencer, D. R. Pettit, E. Garcia, and D. C. Shaw, J. Appl. Phys. 70, 3572 (1991).

${ }^{4}$ P. M. Halleck and J. Wackerle, J. Appl. Phys. 47, 976 (1976).

${ }^{5}$ C. S. Yoo, N. C. Holmes, P. C. Souers, C. J. Wu, F. H. Ree, and J. J. Dick, J. Appl. Phys. 88, 70 (2000).

${ }^{6}$ L. Soulard, Ph. D. thesis, L'Universite de Haute-Alsacc, 1990.

${ }^{7}$ L. Soulard and F. Bauer, in Shock Compression of Condensed Matter-1989, edited by S. C. Schmidt, J. N. Johnson, and L. W. Davison (Elsevier, Amsterdam, 1990), p. 817.

${ }^{8}$ V. K. Jindal and D. D. Dlott, J. Appl. Phys. 83, 5203 (1998).

${ }^{9}$ Y. A. Gruzdkov and Y. M. Gupta, J. Phys. Chem. A 104, 11169 (2000).

${ }^{10} \mathrm{P}$. W. Cooper and S. R. Kurowski, Introduction to the Technology of Explosives (VCH, New York, 1996).

${ }^{11} \mathrm{~J}$. Akhavan, The Chemistry of Explosives (Royal Society of Chemistry, Cambridge, England, 2004).

${ }^{12}$ A. D. Booth and F. J. Llewellyn, J. Chem. Soc. 1947, 837.

${ }^{13}$ J. Trotter, Acta Crystallogr. 16, 698 (1963).

${ }^{14}$ H. H. Cady and A. C. Larson, Acta Crystallogr. B 31, 1864 (1975).

${ }^{15}$ O. Tschauner, B. Kiefer, Y. Lee, M. Pravica, M. Nicol, and E. Kim, J. Chem. Phys. 127, 094502 (2007).

${ }^{16}$ B. Olinger, P. M. Halleck, and H. H. Cady, J. Chem. Phys. 62, 4480 (1975).

${ }^{17}$ M. M. Kuklja, F. J. Zerilli, and S. M. Peiris, J. Chem. Phys. 118, 11073 (2003).

${ }^{18}$ E. F. C. Byrd, G. E. Scuseria, and C. F. Chabalowski, J. Phys. Chem. B 108, 13100 (2004).

${ }^{19}$ C. K. Gan, T. D. Sewell, and M. Challacombe, Phys. Rev. B 69, 035116 (2004).

${ }^{20}$ H. V. Brand, J. Phys. Chem. B 109, 13668 (2005).

${ }^{21}$ M. M. Kuklja, S. N. Rashkeev, and F. J. Zerilli, Appl. Phys. Lett.
89, 071904 (2006).

${ }^{22}$ H. Liu, J. J. Zhao, D. Q. Wei, and Z. Z. Gong, J. Chem. Phys. 124, 124501 (2006).

${ }^{23}$ F. J. Zerilli and M. M. Kuklja, J. Phys. Chem. A 110, 5173 (2006).

${ }^{24}$ E. F. C. Byrd and B. M. Rice, J. Phys. Chem. C 111, 2787 (2007).

${ }^{25}$ F. J. Zerilli, J. P. Hooper, and M. M. Kuklja, J. Chem. Phys. 126, 114701 (2007).

${ }^{26}$ F. J. Zerilli and M. M. Kuklja, J. Phys. Chem. A 111, 1721 (2007).

${ }^{27}$ W. F. Perger, S. Vutukuri, Z. A. Dreger, Y. M. Gupta, and K. Flurchick, Chem. Phys. Lett. 422, 397 (2006).

${ }^{28}$ W. F. Perger, J. J. Zhao, J. M. Winey, and Y. M. Gupta, Chem. Phys. Lett. 428, 394 (2006).

${ }^{29}$ W. F. Perger, R. Pandey, M. A. Blanco, and J. J. Zhao, Chem. Phys. Lett. 388, 175 (2004).

${ }^{30}$ P. Hohenberg and W. Hohn, Phys. Rev. 136, B864 (1964); W. Kohn and L. J. Sham, ibid. 140, A1133 (1965).

${ }^{31}$ G. Kresse and J. Furthmuller, Phys. Rev. B 54, 11169 (1996).

${ }^{32}$ G. Kresse and J. Furthmuller, Comput. Mater. Sci. 6, 15 (1996).

${ }^{33}$ J. P. Perdew, J. A. Chevary, S. H. Vosko, K. A. Jackson, M. R. Pederson, D. J. Singh, and C. Fiolhais, Phys. Rev. B 46, 6671 (1992).

${ }^{34}$ J. P. Perdew, J. A. Chevary, S. H. Vosko, K. A. Jackson, M. R. Pederson, D. J. Singh, and C. Fiolais, Phys. Rev. B 48, 4978 (1993).

${ }^{35}$ J. P. Perdew, K. Burke, and M. Ernzerhof, Phys. Rev. Lett. 77, 3865 (1996).

${ }^{36}$ J. P. Perdew, K. Burke, and M. Ernzerhof, Phys. Rev. Lett. 78, 1396 (1997).

${ }^{37}$ D. Vanderbilt, Phys. Rev. B 41, 7892 (1990).

${ }^{38}$ G. Kresse and J. Hafner, J. Phys.: Condens. Matter 6, 8245 (1994).

${ }^{39}$ P. E. Blochl, Phys. Rev. B 50, 17953 (1994). 
${ }^{40}$ G. Kresse and D. Joubert, Phys. Rev. B 59, 1758 (1999).

${ }^{41}$ H. J. Monkhorst and J. D. Pack, Phys. Rev. B 13, 5188 (1976).

${ }^{42}$ L. G. Green and E. L. Lee, in Proceedings of the 13th International Detonation Symposium, edited by S.M. Peiris (Office of Naval Research, Norfolk, VA, 2006), p. 1144.

${ }^{43}$ C. E. Morris, in Sixth Symposium (International) on Detonation, edited by J. Short (Office of Naval Research, Arlington, VA, 1976), p. 396.

${ }^{44}$ J. M. Winey and Y. M. Gupta, J. Appl. Phys. 90, 1669 (2001).

${ }^{45}$ H. V. Brand, Chem. Phys. Lett. 418, 428 (2006).

${ }^{46}$ S. V. Zybin, L. Zhang, H. Kim, A. C. T. van Duin, and W. A.
Goddard, in Proceedings of the 13th International Detonation Symposium, edited by S.M. Peiris (Office of Naval Research, Norfolk, VA, 2006), p. 848.

${ }^{47}$ M. Conroy, I. I. Oleynik, S. V. Zybin, and C. T. White, in Proceedings of the 13th International Detonation Symposium, edited by S.M. Peiris (Office of Naval Research, Norfolk, VA, 2006), p. 1191.

${ }^{48}$ M. Conroy, I. I. Oleynik, S. V. Zybin, and C. T. White, in Shock Compression of Condensed Matter - 2007, edited by M. Elert, M. D. Furnish, R. Chau, N. Holmes, and J. Nguyen, AIP Conf. Proc. No. 955 (AIP, Melville, 2007), p. 361. 